\title{
COMMUTATIVE ALGEBRA IN STABLE HOMOTOPY THEORY AND A COMPLETION THEOREM
}

\author{
A. D. Elmendorf, J. P. C. Greenlees, I. Kriz, and J. P. May
}

\begin{abstract}
We construct a category of spectra that has all limits and colimits and also has a strictly associative and commutative smash product. This provides the ground category for a new theory of structured ring and module spectra that allows the wholesale importation of techniques of commutative algebra into stable homotopy theory. Applications include new constructions of basic spectra, new generalized universal coefficient and Künneth spectral sequences, and a new construction of topological Hochschild homology. The theory works equivariantly, where it allows the construction of equivariant versions of Brown-Peterson, Morava $K$-theory, and other module spectra over $M U$. Via a topological realization of "local homology and cohomology groups", the general theory leads to a completion theorem for the computation of $M_{*}(B G)$ and $M^{*}(B G)$ in terms of equivariant cobordism groups, where $M$ is $M U, B P, k(n), K(n)$, or any other module spectrum over $M U$. (The reader most interested in the equivariant applications may wish to read the last section first.)
\end{abstract}

\section{Background on the stable homotopy category}

Stable homotopy theory embraces homology and cohomology theory and is a major branch of algebraic topology. Over thirty years ago, it became apparent that the appropriate setting in which to study stable phenomena is the "stable homotopy category" of "spectra" (or "stable spaces"). The translation from topology to algebra through such tools as the Adams spectral sequence becomes far smoother and more structured when carried out in this category, and many constructions and arguments that are essential to calculations are impossible to carry out without it.

The stable homotopy category is "triangulated", which means that it has a suspension functor that is an equivalence of categories together with exact triangles, or cofibration sequences, that satisfy appropriate axioms.

1991 Mathematics Subject Classification. Primary: 55N20, 55N22, 55N91, 55P42, 55P91, 55T25; Secondary: 13D45, 19L47.

The second author acknowledges support from the Nuffield Foundation, the third author is an Alfred P. Sloan Fellow, and the third and fourth authors acknowledge support from the NSF. 
It also has a "smash product", denoted $\wedge$, that is associative, commutative, and unital up to coherent natural isomorphisms, the unit being the sphere spectrum $S$. Abstractly, a category with such a product is said to be "symmetric monoidal" (or "tensored"). This structure allows one to transport algebraic notions such as ring and module into stable homotopy theory. Thus, in the stable homotopy category of spectra - which we denote by $\bar{h} \mathscr{S}$ - a ring is a spectrum $R$ together with a product $\phi: R \wedge R \rightarrow R$ and unit $\eta: S \rightarrow R$ such that the following diagrams commute in $\bar{h} \mathscr{S}$ :

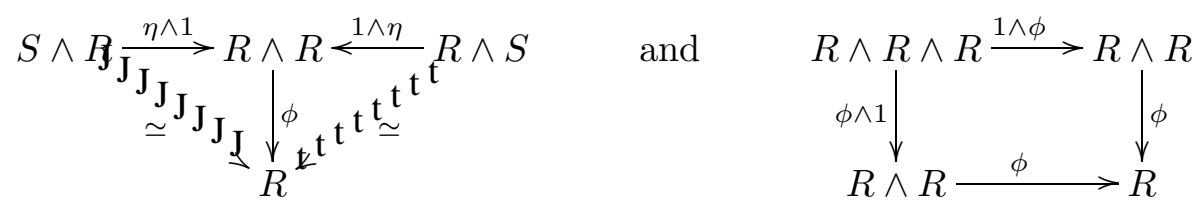

The unlabelled equivalences are canonical isomorphisms in $\bar{h} \mathscr{S}$ that give the unital property, and we have suppressed such an associativity isomorphism in the second diagram. Intuitively, these diagrams commute only up to homotopy. Similarly, there is a transposition isomorphism $\tau$ : $E \wedge F \rightarrow F \wedge E$ in $\bar{h} \mathscr{S}$, and $R$ is commutative if the following diagram commutes in $\bar{h} \mathscr{S}$ :

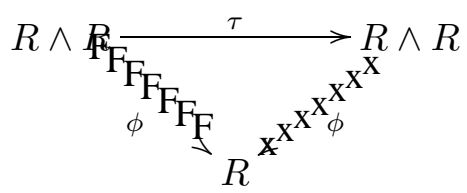

An $R$-module is a spectrum $M$ together with a map $\mu: R \wedge M \rightarrow M$ such that the following diagrams commute in $\bar{h} \mathscr{S}$ :
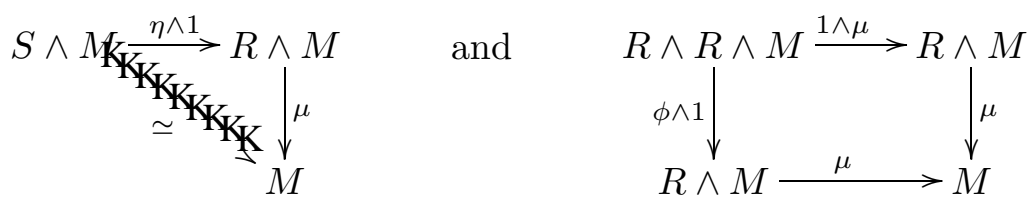

The stable homotopy category admits many different constructions. They are obtained, essentially, by passage to homotopy categories from inequivalent point-set level categories of spectra, e.g. [4, 2, 16, 9]. However, all known point-set level precursors of the stable homotopy category suffer from one defect or another. In fact, it has long been accepted wisdom that there can be no construction of the stable homotopy category from an underlying category of spectra that is both complete and cocomplete 
(has arbitrary limits and colimits) and has an associative and commutative smash product.

Over twenty years ago, it became apparent that it would be of great value to have more precisely structured point-set level notions of ring and module, with good properties before passage to homotopy. May, Quinn, and Ray [20] defined " $A_{\infty}$ and $E_{\infty}$ ring spectra" in 1972. They used "operad actions" defined in terms of "twisted half-smash products" to compensate for the lack of an associative and commutative smash product. In the early 1980's, such rings up to homotopy were advertised by Waldhausen under the rubric "brave new rings." Elmendorf and May [unpublished] and Robinson [22] defined " $A_{\infty}$ and $E_{\infty}$ module spectra" in 1983. The $A_{\infty}$ notions give the associative theory and have been studied by Robinson $[22,23,24,25]$. The $E_{\infty}$ notions add commutativity.

Some examples of $E_{\infty}$ ring spectra are the sphere spectrum $S$, the Eilenberg-MacLane spectrum $H R$ associated to a commutative ring $R$, the Thom spectra $M O$ and $M U$, the spectra $k O$ and $k U$ that represent connective real and complex $K$-theory, and the algebraic $K$-theory spectrum $K R$ associated to a commutative ring $R[20,21,16]$. Recent work of Hopkins, Miller, McClure, Vogt, Schwänzl, and ourselves gives many more examples. In particular, the third author has proven that the BrownPeterson spectra $B P$ are $E_{\infty}$ ring spectra.

While $E_{\infty}$ ring spectra have been studied extensively, ours is the first study of $E_{\infty}$ modules. This point-set level notion of module is far more powerful than the up to homotopy notion given above. Working in $\bar{h} \mathscr{S}$, it is not even true that the cofiber of a map of $R$-modules is an $R$-module, so that one does not have a triangulated category of $R$-modules. Much more deeply, when $R$ is commutative, one wants a smash product over $R$, $M \wedge_{R} N$, that is again an $R$-module. This is clearly impossible with the homotopy level definition.

\section{Commutative algebra in stable homotopy theory}

We have a new approach to these algebraic structures. We begin with the category $\mathscr{S}$ of "coordinate free spectra" of $[20,16]$. That category is complete and cocomplete; it has many homotopy equivalent smash products, none of which are associative and commutative. Our first theorem singles out a new category $S \mathscr{M}$ of "S-module spectra" whose objects are spectra in $\mathscr{S}$ with extra structure.

Theorem 1. There is a complete and cocomplete category SM $\mathscr{M}$ of $S$ module spectra that admits an associative and commutative smash product 
$M \wedge_{S} N$ with a natural unit equivalence $\xi: S \wedge_{S} M \rightarrow M$. It also admits function $S$-modules $F_{S}(M, N)$ such that

$$
S \mathscr{M}\left(L \wedge_{S} M, N\right) \cong S \mathscr{M}\left(L, F_{S}(M, N)\right) .
$$

The map $\xi$ satisfies the expected properties of an action of $S$ on $M$, hence the term " $S$-module". Replacing $\wedge$ by $\wedge_{S}$ in the definitions of ring and module spectra above, it now makes sense to require the displayed diagrams to commute on the point-set level, that is, to commute in the category $S \mathscr{M}$. We thereby obtain new definitions of ring and module spectra. The new notion of a ring spectrum can be given a more conceptual formulation. There is a modified version of the smash product, $M *_{S} N$, that is defined on spectra equipped with given "unit maps" $S \rightarrow M$; this smash product is unital, and there results a symmetric monoidal category $S \mathscr{M}_{u}$ of $S$-modules with unit. Our new definition of a (commutative) ring spectrum coincides with the obvious notion of a (commutative) monoid in this symmetric monoidal category. Remarkably, these new definitions coincide exactly with the original, and seemingly much more complicated, definitions of $A_{\infty}$ and $E_{\infty}$ ring and module spectra.

These reinterpretations of the definitions of structured ring and module spectra place them in a far more technically manageable framework. This conceptual simplicity allows easy versions of constructions that before were difficult or seemingly impossible. Our work on structured ring spectra overlaps ongoing work of Hopkins, Miller, and McClure. In particular, the following sample result — whose proof is now a formal triviality — has been emphasized by Hopkins.

Proposition 2. If $R$ and $R^{\prime}$ are $E_{\infty}$ ring spectra, then $R *_{S} R^{\prime}$ is an $E_{\infty}$ ring spectrum and is the coproduct of $R$ and $R^{\prime}$ in the category of $E_{\infty}$ ring spectra. If $M$ is an $R$-module and $M^{\prime}$ is an $R^{\prime}$-module, then $M \wedge_{S} M^{\prime}$ is an $R *_{S} R^{\prime}$-module.

An $A_{\infty}$ ring spectrum $R$ is analogous to an algebra over a commutative ring $k$, and, exactly as in algebra, we can define the smash product over $R$ of a right $R$-module $M$ and a left $R$-module $N$ to be the coequalizer of the pair of maps

$$
\mu \wedge 1,1 \wedge \nu: M \wedge_{S} R \wedge_{S} N \rightarrow M \wedge_{S} N
$$

where $\mu: M \wedge_{S} R \rightarrow M$ and $\nu: R \wedge_{S} N \rightarrow N$ are the given actions. Similarly, for left $R$-modules $M$ and $N$, there is a function $S$-module $F_{R}(M, N)$; it is defined as the equalizer of an easily defined pair of maps

$$
F_{S}(M, N) \rightarrow F_{S}\left(R \wedge_{S} M, N\right)
$$


Specializing to $E_{\infty}$ ring spectra, we can generalize Theorem 1.

Theorem 3. Let $R$ be an $E_{\infty}$ ring spectrum. The category $R \mathscr{M}$ of $R$ module spectra is complete and cocomplete. It admits an associative and commutative smash product $M \wedge_{R} N$, and there is a natural unit equivalence $\xi: R \wedge_{R} M \rightarrow M$. It also admits function $R$-modules $F_{R}(M, N)$ such that

$$
R \mathscr{M}\left(L \wedge_{R} M, N\right) \cong R \mathscr{M}\left(L, F_{R}(M, N)\right) .
$$

Further, there is an associative composition pairing

$$
F_{R}(M, N) \wedge_{R} F_{R}(L, M) \rightarrow F_{R}(L, N) .
$$

There is also a theory of left-right bimodules with all of the properties that one would expect. In the $A_{\infty}$ context, it specializes to recover the results of $[22,24,25]$.

Again, there is a modified smash product, $M *_{R} N$, of $R$-modules with unit maps $R \rightarrow M$, and there results a symmetric monoidal category $R \mathscr{M}_{u}$ of unital $R$-modules. A monoid in this category is an " $A_{\infty} R$-algebra". For any $R$-module $M, F_{R}(M, M)$ is such an $R$-algebra. Many other standard algebraic concepts translate similarly into our topological context.

The stable homotopy category $\bar{h} \mathscr{S}$ is constructed from the homotopy category of spectra by adjoining formal inverses to the weak equivalences. Analogously, we construct the stable homotopy category $\bar{h} R \mathscr{M}$ of $R$ modules from the homotopy category of $R$-modules by adjoining formal inverses to the weak equivalences, where a weak equivalence is a map of $R$-modules that is a weak equivalence of underlying spectra. The category $\bar{h} S \mathscr{M}$ is our new model for the stable homotopy category.

Theorem 4. The forgetful functor $S \mathscr{M} \rightarrow \mathscr{S}$ induces an equivalence of categories $\bar{h} S \mathscr{M} \rightarrow \bar{h} \mathscr{S}$. For $S$-modules $M$ and $N$, there are natural isomorphisms in $\bar{h} \mathscr{S}$

$$
M \wedge N \simeq M \wedge_{S} N \text { and } F(M, N) \simeq F_{S}(M, N) .
$$

The categories $\bar{h} R \mathscr{M}$ provide a powerful new tool in stable homotopy theory. They are also of considerable intrinsic interest, as the following result illustrates.

Theorem 5. Let $R$ be a commutative ring. Then $R$-modules $M$ can be realized functorially by Eilenberg-Mac Lane spectra $H M$ that are modules over the $E_{\infty}$ ring spectrum $H R$, and

$\operatorname{Tor}_{n}^{R}(M, N) \cong \pi_{n}\left(H M \wedge_{H R} H N\right)$ and $\operatorname{Ext}_{R}^{n}(M, N) \cong \pi_{-n} F_{H R}(H M, H N)$ 
as $R$-modules. Further, the stable homotopy category of HR-modules is equivalent to the derived category of $R$-modules.

The essential point is that our $H M \wedge_{H R} H N$ and $F_{H R}(H M, H N)$ are equivalent to derived tensor product and Hom functors in the category of chain complexes of $R$-modules. The $A_{\infty}$ analog of this theorem recovers the results of [23].

Another interesting example is the stable homotopy category $\bar{h} K(F) \mathscr{M}$ of the algebraic $K$-theory $E_{\infty}$ ring spectra $K(F)$ associated to a field $F$. As noted at the end of [13], this category gives a possible home for the integral mixed Tate motives of $F$ in algebraic geometry.

For any $E_{\infty}$ ring spectrum $R$, there is a free $R$-module functor $F$ : $\mathscr{S} \rightarrow R \mathscr{M}$. Taking "sphere $R$-modules" to be $R$-modules of the form $F S^{n}$, we develop a theory of cell and CW $R$-modules that is exactly like the usual theory of cell and CW spectra. A weak equivalence of cell $R$ modules is a homotopy equivalence, any $R$-module is weakly equivalent to a cell $R$-module, and $\bar{h} R \mathscr{M}$ is equivalent to the homotopy category of cell $R$-modules. Functors on $R$-modules that do not preserve weak equivalences are transported to the category $\bar{h} R \mathscr{M}$ by first approximating their arguments by cell $R$-modules; the last statement of Theorem 4 must be interpreted in this light.

For a spectrum $E$, let $E_{n}=\pi_{n}(E)=E^{-n}$. By using free $R$-modules to realize free $R_{*}$-module resolutions of $M_{*}$ topologically, we obtain the following calculational generalization of Theorem 5 . It gives a kind of Eilenberg-Moore (or hyperhomology) spectral sequence in stable homotopy theory.

Theorem 6. Let $R$ be an $E_{\infty}$ ring spectrum. For $R$-modules $M$ and $N$, there are spectral sequences of differential $R_{*}$-modules

$\operatorname{Tor}_{*, *}^{R_{*}}\left(M_{*}, N_{*}\right) \Longrightarrow \pi_{*}\left(M \wedge_{R} N\right)$ and $\operatorname{Ext}_{R_{*}^{* * *}}^{*}\left(M_{*}, N^{*}\right) \Longrightarrow \pi_{-*}\left(F_{R}(M, N)\right)$.

These give universal coefficient and Künneth spectral sequences by specialization.

Theorem 7 (universal coefficient). For an $R$-module $N$ and an $S$-module $X$, there are spectral sequences of differential $R_{*}$-modules

$$
\operatorname{Tor}_{*, *}^{R_{*}}\left(R_{*} X, N_{*}\right) \Longrightarrow N_{*} X \text { and } \operatorname{Ext}_{R_{*}^{* *}}^{*}\left(R_{*} X, N^{*}\right) \Longrightarrow N^{*} X .
$$


Theorem 8 (Künneth). For $S$-modules $X$ and $Y$, there are spectral sequences of differential $R_{*}$-modules

$\operatorname{Tor}_{*, *}^{R_{*}}\left(R_{*} X, R_{*} Y\right) \Longrightarrow R_{*}(X \wedge Y)$ and $\operatorname{Ext}_{R_{*}^{* * *}}^{*}\left(R_{*} X, R^{*} Y\right) \Longrightarrow R^{*}(X \wedge Y)$.

There are spectral sequences more generally when $R$ is only an $A_{\infty}$ ring spectrum, but without the $R_{*}$-module structure needed for explicit calculation. Such spectral sequences were first obtained by Robinson [22, 24]. Adams [1] derived universal coefficient and Künneth spectral sequences like these under ad hoc calculational hypotheses that require case-by-case verification.

We can realize many other homological constructions topologically. A particularly interesting example is a generalization of Bökstedt's topological Hochschild homology $T H H(A)$ [5] from discrete rings $A$ to $A_{\infty}$ algebras over $E_{\infty}$ ring spectra. The Hochschild homology groups of an algebra $A$ over a commutative ring $R$ can be computed as the homology groups of the chain complex of $R$-modules associated to a certain simplicial R-module $H_{\bullet}(A)$ such that $H_{n}(A)$ is the $n$-fold tensor power of $A$; the faces and degeneracies of $H_{\bullet}(A)$ are defined in terms of the product and unit of $A$. If $A$ is commutative, then $H_{\bullet}(A)$ is a simplicial $R$-algebra.

Now let $R$ be an $E_{\infty}$ ring spectrum and let $A$ be an $A_{\infty} R$-algebra. Simply by replacing tensor products over $R$ by smash products $*_{R}$ in the explicit definition of $H_{\bullet}(A)$, we obtain a simplicial spectrum $H_{\bullet}(A)$ whose geometric realization we call $T H H(A)$. If $A$ is an $E_{\infty} R$-algebra, then $H_{\bullet}(A)$ is a simplicial $E_{\infty} R$-algebra and $T H H(A)$ is an $E_{\infty} R$-algebra. Its homotopy groups are the topological Hochschild homology groups of $A$ (relative to $R$ ). In fact, $H_{\bullet}(A)$ is a cyclic $R$-module spectrum and $T H H(A)$ has an action of the circle group $S^{1}$. It seems that a slightly more sophisticated construction gives a better version of the $S^{1}$-action that makes $T H H(A)$ a cyclotomic spectrum in the sense of [19], and in fact a cyclotomic $R$-module. Assuming this, we will also have a generalization of topological cyclic homology from discrete rings to $A_{\infty}$ algebras over $E_{\infty}$ ring spectra. However, this is work in progress.

Another immediate application of our general theory gives a homotopytheoretic replacement for the Baas-Sullivan theory of manifolds with singularities $[3,6]$. The latter theory uses geometric methods to construct spectra by killing or inverting generators in the homotopy groups of certain cobordism spectra, especially $M U$. Recent work in stable homotopy theory has focused on the study of these fundamentally important spectra, but their manifold-theoretic construction bears negligible relation to the techniques of their study and has made it difficult to analyze their multiplicative and uniqueness properties. Moreover, we can now obtain 
equivariant versions of all of these spectra, something that would be extremely difficult, if it could be done at all, using manifolds with singularities. We shall return to $M U$ shortly. The basic construction is applicable to modules over any $E_{\infty}$ ring spectrum.

Construction 9. Let $R$ be an $E_{\infty}$ ring spectrum, let $x \in \pi_{n}(R)$, and let $M$ be an $R$-module. Then we can realize the map on homotopy groups given by multiplication by $x$,

$$
\cdot x: \pi_{*} M \rightarrow \pi_{*} M,
$$

as the map on homotopy groups induced by the map of $R$-modules

$$
\mu \circ\left(x \wedge_{S} 1\right): S^{n} \wedge_{S} M \rightarrow R \wedge_{S} M \rightarrow M .
$$

The cofiber $M / x M$ of this map is again an $R$-module. We can therefore iterate and pass to telescopes to construct $M / X M$ for any sequence $X=$ $\left\{x_{1}, \ldots, x_{i}, \ldots\right\}$ of elements of $\pi_{*} R$. If $I$ is the ideal generated by $X$ and if $\mathrm{X}$ is a regular sequence for $\pi_{*} M$, then the resulting map $M \rightarrow M / X M$ induces an isomorphism

$$
\pi_{*}(M) / I \pi_{*}(M) \cong \pi_{*}(M / X M) .
$$

Similarly, we can use iterated multiplication by elements and passage to telescopes to construct an $R$-module $M\left[X^{-1}\right]$ and a map $M \rightarrow M\left[X^{-1}\right]$ that induces localization

$$
\pi_{*}(M) \rightarrow \pi_{*}(M)\left[X^{-1}\right] .
$$

Techniques of conventional stable homotopy theory in the stable homotopy category of $R$-modules can be used to derive information about the multiplicative structures on spectra constructed in this fashion. In particular, all of the known information about the multiplicative structure on the spectra usually constructed by Baas-Sullivan theory can be obtained in this way. We can also construct completions of modules at ideals in $\pi_{*}(R)$, as we explain in an equivariant setting.

\section{New equivariant spectra and a completion theorem}

Completion theorems relate the nonequivariant cohomology of classifying spaces to algebraic completions of associated equivariant cohomology theories. They are at the heart of equivariant stable homotopy theory and its nonequivariant applications. Two fundamental theorems along this line are the Atiyah-Segal completion theorem and the Segal conjecture. The 
first asserts that, for any compact Lie group $G, K^{*}(B G)$ is the completion of $K_{G}^{*} \equiv K_{G}^{*}(p t)$ at the augmentation ideal of the representation ring $R(G)$. Here $K_{G}^{0}=R(G), K_{G}^{1}=0$, and $K_{G}^{*}$ is periodic with period 2 . The second asserts that, for any finite group $G$, the stable cohomotopy $\pi^{*}(B G)$ is isomorphic to the completion of $\pi_{G}^{*}$ at the augmentation ideal of the Burnside ring $A(G)$. Here $\pi_{G}^{0}=A(G), \pi_{G}^{q}=0$ for $q>0$, and the $\pi_{G}^{q}$ for $q<0$ are unknown in general. Thus the Segal conjecture proves the equality of two interesting sequences of invariants of the group $G$, both of which are very hard to compute.

Let $G$ be a compact Lie group. Our general theory of structured ring and module spectra applies verbatim to $G$-spectra and allows us to construct new equivariant spectra and to prove completion theorems about them. Two problems stated and discussed by Carlsson in his recent survey [7] of equivariant stable homotopy theory read as follows:

"Define and compute equivariant Morava K-theory spectra."

"Formulate a conjecture about $M U^{*}(B G)$, for $G$ a finite group."

The second problem had already been posed by Landweber in 1970 [14], and he noted that the problem of studying $M U_{*}(B G)$ seemed to be even harder. Prior to our work, nothing at all was known about the first problem and, except when $G$ is Abelian, little was known about the second. On the conceptual level, we shall solve both problems; on the computational level, however, our work will merely point the direction to further study.

There is a stabilized version of equivariant cobordism that was introduced by tom Dieck [8]. It is represented by an $E_{\infty}$ ring $G$-spectrum $M U_{G}$ with underlying nonequivariant $E_{\infty}$ ring spectrum $M U$. The $G$ spectrum $M U_{G}$ is "split," which means that there is a map from $M U$ to the fixed point spectrum $\left(M U_{G}\right)^{G}$ whose composite with the inclusion $\left(M U_{G}\right)^{G} \rightarrow M U$ is the identity. Therefore $M U_{*}=\pi_{*}(M U)$ is a direct summand of $M U_{*}^{G} \equiv \pi_{*}^{G}\left(M U_{G}\right)$. This allows us to construct equivariant versions of the Brown-Peterson spectrum $B P$, the Morava $K$-theory spectra $k(n)$ and $K(n)$, and all of the other spectra that are usually constructed from $M U$ by means of the Baas-Sullivan theory of manifolds with singularities.

Recall that $\pi_{*}(M U)=\mathbb{Z}\left[x_{i} \mid \operatorname{deg}\left(x_{i}\right)=2 i\right]$. Starting from $M U_{G}$, we can construct $M U_{G}$-module spectra by killing off any chosen sequence of elements of $\pi_{*}(M U)$ and inverting any other chosen sequence. If we kill off the $x_{i}$ for $i$ not of the form $p^{n}-1$ and then localize at $p$, we obtain the Brown-Peterson spectrum $B P_{G}$. Its underlying nonequivariant spectrum is $B P$. Recall that $\pi_{*}(B P)=\mathbb{Z}_{(p)}\left[v_{i} \mid \operatorname{deg}\left(v_{i}\right)=2\left(p^{i}-1\right)\right]$, where the generators $v_{i}$ come from $\pi_{*}(M U)$ (provided that we use the Hazewinkel 
generators). We list a few of the important spectra derived from $B P$, with their coefficient rings. Let $\mathbb{F}_{p}$ denote the field with $p$ elements.

$$
\begin{array}{llll}
B P\langle n\rangle & \mathbb{Z}_{(p)}\left[v_{1}, \ldots, v_{n}\right] & E(n) & \mathbb{Z}_{(p)}\left[v_{1}, \ldots, v_{n}, v_{n}^{-1}\right] \\
P(n) & \mathbb{F}_{p}\left[v_{n}, v_{n+1}, \ldots\right] & B(n) & \mathbb{F}_{p}\left[v_{n}^{-1}, v_{n}, v_{n+1}, \ldots\right] \\
k(n) & \mathbb{F}_{p}\left[v_{n}\right] & K(n) & \mathbb{F}_{p}\left[v_{n}, v_{n}^{-1}\right]
\end{array}
$$

We construct $B P\langle n\rangle_{G}$ from $B P_{G}$ by killing off the $v_{i}$ for $i>n$, and we construct $E(n)_{G}$ from $B P\langle n\rangle_{G}$ by inverting $v_{n}$. We construct $k(n)_{G}$ and $K(n)_{G}$ from $B P\langle n\rangle_{G}$ and $E(n)_{G}$ by killing off the $v_{i}$ for $i<n$. We do not know that the sequences of elements that we are killing are regular sequences in $M U_{*}^{G}$, but that does not affect the constructions. The constructions are independent of the order in which we kill and invert elements. It is immediate that the underlying nonequivariant spectra of $B P\langle n\rangle_{G}, k(n)_{G}, K(n)_{G}$, etc, are $B P\langle n\rangle, k(n), K(n)$, etc, and the $G$ spectra constructed in this fashion are split. It is also immediate that our constructions are functorial in $G$. These are two of the primary desiderata of equivariant Brown-Peterson and Morava $K$-theory $G$-spectra. Explicit computations will require better understanding of $M U_{*}^{G}$ than is now available. We give a perhaps over-optimistic conjecture.

Conjecture 10. $M U_{*}^{G}$ is $M U_{*}$-free on generators of even degree.

This was stated by Löffler $[17,18]$ when $G$ is Abelian, but his proof has not appeared. Comezana (unpublished) has recently supplied a complete argument. Again, little is known for non-Abelian groups.

A third desideratum of equivariant Morava $K$-theories is that there should be a close relationship between $K(n)_{G}^{*}$ and $K(n)^{*}(B G)$. We have a surprisingly general theorem along these lines: it is valid for a large class of groups $G$ and for all $M U_{G}$-module spectra $M$ (in the $E_{\infty}$ sense), and it deals with $M_{*}(B G)$ as well as $M^{*}(B G)$. It shows that these homology and cohomology groups are the homotopy groups of certain $G$-spectra $H_{I}\left(M U_{G} ; M\right)$ and $H^{I}\left(M U_{G} ; M\right)$. There are spectral sequences for the computation of these groups in terms of "local cohomology groups" and "local homology groups" that can be computed from knowledge of the ring $M U_{*}^{G}$. Thus it establishes a close connection between the geometrically defined equivariant cobordism groups and the homology and cohomology of classifying spaces with coefficients in $M U$-module spectra.

The idea is to mimic the second author's homological version of the Atiyah-Segal completion theorem [10], using our new technology. This entails the importation of techniques of commutative algebra into equivariant stable homotopy theory, and we must set out briefly the relevant constructions from commutative algebra. Let $R$ be a graded commutative 
ring and let $I=\left(\alpha_{1}, \ldots, \alpha_{n}\right)$ be a finitely generated ideal in $R$. Define $K^{\bullet}(I)$ to be the tensor product of the graded chain complexes

$$
K^{\bullet}\left(\alpha_{i}\right)=\left(R \rightarrow R\left[1 / \alpha_{i}\right]\right)
$$

where $R$ and $R\left[1 / \alpha_{i}\right]$ lie in homological degrees 0 and 1 . Up to quasiisomorphism, $K^{\bullet}(I)$ depends only on the radical of $I$. For a graded $R$ module $M$, define

$$
H_{I}^{s, t}(R ; M)=H^{s, t}\left(K^{\bullet}(I) \otimes M\right),
$$

where $s$ indicates the homological degree and $t$ the internal grading. Such "local cohomology groups" were first defined by Grothendieck. Special cases of these algebraic local cohomology groups turn out to compute topological homology groups.

There are dual "local homology groups" which, to the best of our knowledge, were first introduced in $[11,12]$. Replacing $K^{\bullet}(I)$ by a quasiisomorphic $R$-free chain complex $K^{\prime \bullet}(I)$, define

$$
H_{s, t}^{I}(R ; M)=H_{s, t}\left(\operatorname{Hom}\left(K^{\prime \bullet}(R), M\right)\right) .
$$

There is a tri-graded universal coefficient spectral sequence converging to these groups; ignoring the internal grading $t$, which is unchanged by the differentials, it converges in total degree $s=-(p+q)$ and satisfies

$$
E_{2}^{p, q}=\operatorname{Ext}_{R}^{p}\left(H_{I}^{-q}(R ; R), M\right) \text { and } d_{r}: E_{r}^{p, q} \rightarrow E_{r}^{p+r, q-r+1} .
$$

There is a natural epimorphism $H_{0}^{I}(R ; M) \rightarrow M_{I} \hat{\text { whose kernel is a certain }}$ $\lim ^{1}$ group. If $R$ is Noetherian, then $H_{*}^{I}(R ; M)$ calculates the left derived functors of $I$-adic completion [12].

Now let $R$ be an $E_{\infty} \operatorname{ring} G$-spectrum and $M$ be an $E_{\infty} R$-module. For $\alpha \in R_{k}^{G}$, let $R[1 / \alpha]$ be the telescope of iterates

$$
R \rightarrow \Sigma^{-k} R \rightarrow \Sigma^{-2 k} R \rightarrow \cdots
$$

of multiplication by $\alpha$ and let $K(\alpha)$ be the fiber of the canonical map $R \rightarrow R[1 / \alpha]$. For a finitely generated ideal $I=\left(\alpha_{1}, \ldots, \alpha_{n}\right)$ in $R_{*}$, let $K(I)$ be the smash product over $R$ of the $R$-modules $K(\alpha)$. Up to equivalence of $R$-modules, $K(I)$ depends only on the radical of $I$. Define the "local cohomology spectrum of $M$ " to be the $R$-module

$$
H_{I}(R ; M) \equiv K(I) \wedge_{R} M .
$$

Define the "local homology spectrum of $M$," alias the completion of $M$ at $I$, to be the $R$-module

$$
H^{I}(R ; M) \equiv F_{R}(K(I), M) \equiv M_{I} .
$$


There is a spectral sequence converging to $H_{I}(R ; M)_{*}^{G}$ (in total degree $p+q)$, with

$$
E_{p, q}^{2}=H_{I}^{-p,-q}\left(R_{*}^{G} ; M_{*}^{G}\right) \text { and } d^{r}: E_{p, q}^{r} \rightarrow E_{p-r, q+r-1}^{r},
$$

and there is a spectral sequence converging to $H^{I}(R ; M)_{G}^{*}$ (in total degree $p+q)$ with

$$
E_{2}^{p, q}=H_{-p,-q}^{I}\left(R_{G}^{*} ; M_{G}^{*}\right) \text { and } d_{r}: E_{r}^{p, q} \rightarrow E_{r}^{p+r, q-r+1} .
$$

Now take $I$ to be the augmentation ideal, $I=\operatorname{Ker}\left(R_{*}^{G} \rightarrow R_{*}\right)$. We need to know that $I$ is the radical of a finitely generated ideal in order to perform our constructions, but the rings that we are interested in are not Noetherian. We also need a compatibility relation with change of groups to allow induction.

Proposition 11. If $G$ acts freely on a finite product of unit spheres of representations, for example if $G$ is nilpotent, then the augmentation ideal $I=I G$ of $M U_{*}^{G}$ is the radical of a finitely generated ideal. Moreover, for $H \subset G$, the radical of the augmentation ideal $I H$ of $M U_{*}^{H}$ is equal to the radical of the ideal generated by the image of IG under restriction.

The canonical map $K(I) \rightarrow R$ is an equivalence of underlying spectra, and there results a map

$$
\kappa: E G_{+} \wedge R \rightarrow K(I)
$$

of $R$-module spectra over $R$, where $E G_{+}$is the union of $E G$ and a $G$-fixed disjoint basepoint. The map $\kappa$ induces maps of $R$-modules

$$
E G_{+} \wedge M \simeq E G_{+} \wedge R \wedge_{R} M \rightarrow K(I) \wedge_{R} M=H_{I}(R ; M)
$$

and

$$
H^{I}(R ; M)=F_{R}(K(I), M) \rightarrow F_{R}\left(E G_{+} \wedge R, M\right) \simeq F\left(E G_{+}, M\right),
$$

both of which will be equivalences for all $M$ if $\kappa$ is an equivalence. We can now state our completion theorem for modules over $M U_{G}$.

Theorem 12. If $G$ acts freely on a finite product of unit spheres of representations, then $\kappa: E G_{+} \wedge M U_{G} \rightarrow K(I)$ is an equivalence. Therefore,

$$
E G_{+} \wedge M \rightarrow H_{I}\left(M U_{G} ; M\right) \text { and } H^{I}\left(M U_{G} ; M\right) \rightarrow F\left(E G_{+}, M\right)
$$

are equivalences for any $M U_{G}$-module $M$. 
It is valuable to obtain a completion theorem about $E G \times_{G} X$ for a general $G$-space $X$, obtaining the motivating result about $B G$ by taking $X$ to be a point. For this purpose, we replace $M$ by $M \wedge X_{+}$in the first equivalence and by $F\left(X_{+}, M\right)$ in the second. If $\mathrm{M}$ is split, then

$$
\pi_{*}^{G}\left((E G \times X)_{+} \wedge \Sigma^{-A d(G)} M\right) \cong M_{*}\left(E G \times_{G} X\right)
$$

and

$$
\pi_{*}^{G}\left(F\left((E G \times X)_{+}, M\right)\right) \cong M^{*}\left(E G \times_{G} X\right),
$$

where $A d(G)$ is the adjoint representation of $G$ [16, II.8.4]. Thus the theorem has the following immediate consequence.

Theorem 13. If $G$ acts freely on a finite product of unit spheres of representations and $M$ is a split $M U_{G}$-module spectrum, then

$$
H_{I}\left(M U_{G} ; \Sigma^{-A d(G)} M \wedge X_{+}\right)_{*}^{G} \cong M_{*}\left(E G \times_{G} X\right)
$$

and

$$
H^{I}\left(M U_{G} ; F\left(X_{+}, M\right)\right)_{G}^{*} \cong M^{*}\left(E G \times_{G} X\right)
$$

for any $G$-space $X$.

We conjecture that the hypothesis on $G$ can be removed in the previous theorems. More precisely, we conjecture that $K(J)$ is independent of $J$ for sufficiently large finitely generated subideals $J$ of $I$ and that the theorems hold with $I$ replaced by any such ideal $J$. [Added in proof: we have now proven this conjecture for all finite groups $G$.]

As we have indicated, there are spectral sequences for the computation of the groups on the left in terms of local cohomology and local homology groups, respectively. In this connection, we note the following result of Löffler [18].

Theorem 14 (Löffler). If $G$ is a compact Abelian Lie group, then

$$
\left(M U_{G}^{*}\right) \hat{I} \cong M U^{*}(B G) \text {. }
$$

Here $M U^{*}(B G)$ is completely understood $[15,17,18]$. In this case, the topology forces the following algebraic conclusion.

Corollary 15. If $G$ is a compact Abelian Lie group, then

$$
H_{0}^{I}\left(M U_{G}^{*} ; M_{G}^{*}\right) \cong\left(\left(M U_{G}\right)_{\hat{I}}\right)_{G}^{*} \cong\left(M U_{G}^{*}\right) \hat{I}
$$

and

$$
H_{p}^{I}\left(M U_{G}^{*} ; M_{G}^{*}\right)=0 \text { if } p \neq 0 .
$$


Like the Segal conjecture, our Theorem 13 relates two very interesting unknowns: the equivariant stabilized cobordism groups and the nonequivariant cohomology of classifying spaces for theories that are represented by $E_{\infty}$-module spectra over $M U$, such as $M U$ itself and $B P, k(n)$, and $K(n)$. There is a long and extensive history of explicit calculations of groups $M_{*}(B G)$ and $M^{*}(B G)$ in special cases. Our theorem gives a general conceptual framework into which all such computations must fit.

Our new foundations in stable homotopy theory open up many other avenues of exploration. The new ability to mimic algebraic constructions topologically has only begun to be exploited, and there is much work in progress, by ourselves and others. We are very grateful to Mike Hopkins, Haynes Miller, Jim McClure, Rainer Vogt, Roland Schwänzl, and Ib Madsen for sharing their ideas with us.

\section{References}

1. J. F. Adams, Lectures on generalised cohomology, Springer Lecture Notes 99, 1969, $1-138$.

2. J. F. Adams, Stable homotopy and generalized homology, Univ. of Chicago, 1975.

3. N. A. Baas, On bordism theory of manifolds with singularities, Math. Scand. 33 (1973), 279-302.

4. J. M. Boardman, Stable homotopy theory, Mimeographed notes, Warwick University, 1966-70.

5. M. Bökstedt, Topological Hochschild homology, Topology (to appear).

6. B. I. Botvinnik, Manifolds with Singularities and the Adams-Novikov spectral sequence, London Mathematical Society Lecture Note Series 170, Cambridge University Press, 1992.

7. G. Carlsson, A survey of equivariant stable homotopy theory, Topology 31 (1992), $1-27$.

8. T. tom Dieck, Bordism of G-manifolds and integrality theorems, Topology 9 (1970), 345-358.

9. A. D. Elmendorf, The Grassmannian geometry of spectra, J. Pure and Appl. Algebra 54 (1988), 37-94.

10. J. P. C. Greenlees, K-homology of universal spaces and local cohomology of the representation ring, Topology 32 (1993), 295-308.

11. J. P. C. Greenlees and J. P. May, Completions of G-spectra at ideals of the Burnside ring, Proc. Adams Memorial Symposium, Vol II, Cambridge University Press (1992), 145-178.

12. J. P. C. Greenlees and J. P. May, Derived functors of I-adic completion and local homology, J. Algebra 149 (1992), 438-453.

13. I. Kriz and J. P. May, Derived categories and motives, Math. Res. Let. 1 (1994), 87-94.

14. P.S. Landweber, Cobordism and classifying spaces, Proc. Symp. Pure Math. 22 (1971), 125-129.

15. P. S. Landweber, Coherence, flatness and cobordism of classifying spaces, Proc. Adv. Study Inst. Alg. Top. Aarhus, Denmark, 1970, 257-269. 
16. L. G. Lewis, Jr, J. P. May, and M. Steinberger (with contributions by J. E. McClure), Equivariant stable homotopy theory, Springer Lecture Notes 1213, 1986.

17. P. Löffler, Bordismengruppen unitärer Torusmannigfaltigkeiten, Manuscripta Mathematica 12 (1974), 307-327.

18. P. Löffler, Equivariant unitary bordism and classifying spaces, Proc. International Symposium on Topology and its Applications, Budva, Yugoslavia, 1973, 158-160.

19. I. Madsen, The cyclotomic trace in algebraic K-theory, Proc. ECM. Paris, France, 1992.

20. J. P. May (with contributions by F. Quinn, N. Ray, and J. Törnehave), $E_{\infty}$ ring spaces and $E_{\infty}$ ring spectra, Springer Lecture Notes 577, 1977.

21. J. P. May, Multiplicative infinite loop space theory, J. Pure and Applied Algebra 26 (1982), 1-69.

22. A. Robinson, Derived tensor products in stable homotopy theory, Topology 22 (1983), 1-18.

23. A. Robinson, The extraordinary derived category, Math. Z. 196 (1987), 231-238.

24. A. Robinson, Spectra of derived module homomorphisms, Math. Proc. Camb. Phil. Soc. 101 (1987), 249-257.

25. A. Robinson, Composition products in RHom, and ring spectra of derived endomorphisms, Springer Lecture Notes 1370, 1989, 374-386.

Purdue University Calumet, Hammond IN 46323

E-mail address: aelmendo@math.purdue.edu

Department of Pure Mathematics, University of Sheffield, Sheffield S3 7RH, U.K.

E-mail address: j.greenlees@sheffield.ac.uk

University of Chicago, Chicago Il 60637

E-mail address: kriz@math.uchicago.edu,may@math.uchicago.edu 